\title{
Portales de datos abiertos. Metodología de análisis y aplicación a municipios españoles
}

\author{
Open data portals. Methodology of analysis \\ and application to Spanish municipalities
}

\author{
Sonia Royo-Montañés; Alberto Benítez-Gómez
}

Cómo citar este artículo:

Royo-Montañés, Sonia; Benítez-Gómez, Alberto (2019). “Portales de datos abiertos. Metodología de análisis y aplicación a municipios españoles". El profesional de la información, v. 28, n. 6, e280609.

https://doi.org/10.3145/epi.2019.nov.09

Artículo recibido el 10-06-2019

Aceptación definitiva: 25-10-2019

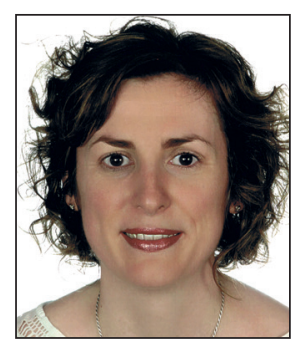

Sonia Royo-Montañés $\bowtie$ https://orcid.org/0000-0001-5162-1446

Universidad de Zaragoza Departamento de Contabilidad y Finanzas

Facultad de Economía y Empresa Gran Vía, 2. 50005 Zaragoza, España sroyo@unizar.es

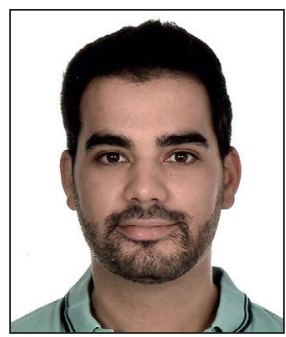

\author{
Alberto Benítez-Gómez \\ https://orcid.org/0000-0002-2924-9550 \\ Universidad de Zaragoza \\ Departamento de Contabilidad y \\ Finanzas \\ Facultad de Economía y Empresa \\ Gran Vía, 2. 50005 Zaragoza, España \\ albertobengom@gmail.com
}

\section{Resumen}

Las administraciones públicas publican datos abiertos para su posterior reutilización por la ciudadanía. Además de un evidente impacto en la transparencia, los datos abiertos tienen un importante potencial económico, lo que explica el auge de las iniciativas para su difusión. Cada vez más administraciones deciden crear un portal de datos abiertos y las entidades locales no se han mantenido al margen de esta tendencia. Pocos trabajos empíricos han evaluado este tipo de portales y los análisis son mayoritariamente de tipo cualitativo o se limitan a un número reducido de portales, por lo que no existen pautas generalmente aceptadas para evaluar su implementación. Por ello, la principal contribución de este trabajo es la definición de una metodología para analizar los portales de datos abiertos, así como su aplicación a una muestra de municipios de España, país que está a la vanguardia en reutilización de datos en Europa. Además, se analiza la información económico-financiera que ofrecen los municipios en sus portales de datos abiertos, por ser fundamental para la rendición de cuentas, la lucha contra la corrupción y la evaluación de la sostenibilidad y eficacia de la prestación de servicios públicos. Los resultados muestran que todavía queda mucho por avanzar en este campo. Sólo un $40 \%$ de las ciudades analizadas cuentan con un portal de datos abiertos y la puntuación media de la muestra analizada no alcanza el $50 \%$. La mayoría de los portales parecen funcionar como simples repositorios de datos, descuidado aspectos que fomentan la utilización de los datos por parte de un público no experto, la rendición de cuentas, la participación ciudadana y la calidad de los datos suministrados (por ejemplo, definición de metadatos utilizados), por lo que se concluye planteando algunas recomendaciones de mejora.

\section{Palabras clave}

Datos abiertos; Portales de datos abiertos; Gobierno abierto; Transparencia; Participación; Colaboración; Reutilización de información del sector público; Gestión pública; Municipios; España.

Agradecimientos

Los autores agradecen a los evaluadores anónimos por sus valiosas sugerencias y acertados comentarios a versiones anteriores de este trabajo.

Financiación

Este trabajo ha sido financiado por los proyectos ECO2015-66240-P Mineco/Feder (Ministerio de Economía y Competitividad, Gobierno de España) S56-17R (Comunidad Autónoma de Aragón/FEDER 2014-2020 Construyendo Europa desde Aragón) y UZ2019-SOC-05 (Universidad de Zaragoza). 


\begin{abstract}
Public administrations publish open data for later reuse by citizens. Besides the obvious impact on transparency, they have an important economic potential which explains the increasing adoption of this type of initiatives. There is an increasing number of administrations that decide to create open data portals, a tendency that municipalities have followed. Few empirical papers have assessed this type of portals and the analyses have been mainly qualitative or limited to a reduced number of portals, so there are no generally accepted criteria for the assessment of their implementation. The main contribution of this paper is the definition of a method to analyse open data portals and its application to a sample of municipalities of Spain, a country at the forefront of data re-utilization in Europe. Furthermore, the economic-financial information available in these portals is analysed, as it is essential to accountability, fight corruption and evaluate the financial sustainability and effectiveness of public service delivery. Results show that there is much room for improvement in this field. Only $40 \%$ of the cities analysed have an open data portal, and the average score of the sample analysed does not reach $50 \%$. Most portals seem to work as mere data repositories, neglecting those aspects that promote data use by the non-expert public, accountability, citizen engagement and the quality of the data being disclosed (e.g., definition of the metadata used). So, the paper concludes with some recommendations for improvement.
\end{abstract}

\title{
Keywords
}

Open data; Open data portals; Open government; Transparency; Participation; Collaboration; Re-use of public sector information; Public sector management; Municipalities; Spain.

\section{Introducción}

Las iniciativas de datos abiertos se enmarcan dentro de los movimientos hacia el gobierno abierto (open government), basado en los pilares de transparencia, participación y colaboración (Obama, 2009; Ferrer-Sapena; Peset; Aleixandre-Benavent, 2011; Chatfield; Reddick, 2017). Aunque esta filosofía de gobierno es relativamente reciente ${ }^{1}$ y no existe una única definición para este fenómeno, de forma resumida podemos indicar que se trata de una forma de gestionar las administraciones que abre la gestión de los asuntos públicos a los ciudadanos, para darles más información y control de las actuaciones de los políticos y gestores públicos y facilitar su participación y colaboración en dicha gestión (Curto-Rodríguez, 2015, p. 66; Medina, 2015, p. 54). Puede distinguirse por tanto una doble vertiente en el gobierno abierto:

1) transparencia y rendición de cuentas y

2) participación ciudadana en la toma de decisiones, diseño de las políticas públicas y co-creación de servicios públicos.

La transparencia es una condición necesaria para lograr la participación y colaboración. En este contexto entran en juego los portales de datos abiertos ${ }^{2}$, como una forma de poner a disposición de todos los agentes sociales los datos que posibilitan los tres objetivos perseguidos por el gobierno abierto (transparencia, participación y colaboración). Por ello las iniciativas de datos abiertos proporcionan la base para lograr la participación y colaboración ciudadana en la creación de servicios públicos innovadores que proporcionen valor añadido (Ubaldi, 2013; Abella; Ortiz-de-Urbina-Criado; De-Pablos-Heredero, 2017b; Cerrillo-Martínez, 2018).

De ahí que algunos autores hablen de estas iniciativas como una forma de gestionar

"que tiene un matiz ético: todo lo que se ha generado con dinero público debería ser accesible para toda la sociedad sin discriminación" (Garriga-Portolà, 2011, p. 299).

Las iniciativas de datos abiertos son cada vez más abundantes. En España, por ejemplo, hace una década que se aprobó la Ley 37/2007, de reutilización de la información del sector público, y desde 2009 el proyecto Aporta y un importante número de iniciativas autonómicas y locales fomentan la apertura de datos de las administraciones públicas y su reutilización por parte de los ciudadanos, situando a España a la vanguardia en reutilización en Europa (Marcos-Martín; Soriano-Maldonado, 2011; Carrara et al., 2015; Carrara; Nieuwenhuis; Vollers, 2016; Berends et al., 2017; Cecconi; Radu, 2018).

Diversos organismos y asociaciones han propuesto recomendaciones para fomentar el uso de datos abiertos y el ámbito científico tampoco se ha quedado al margen, con cada vez más publicaciones sobre este tema. La transversalidad de estas iniciativas es una muestra de su creciente interés, que también ha calado en la administración local (Criado; Ruvalcaba-Gómez, 2016). La constitución de un grupo de trabajo y publicación de una guía estratégica y conjunto de datos mínimos a publicar por parte de la Federación Española de Municipios y Provincias (FEMP, 2017) también da cuenta de la relevancia de estas iniciativas para los municipios. No obstante, algunos autores critican que estos portales parecen

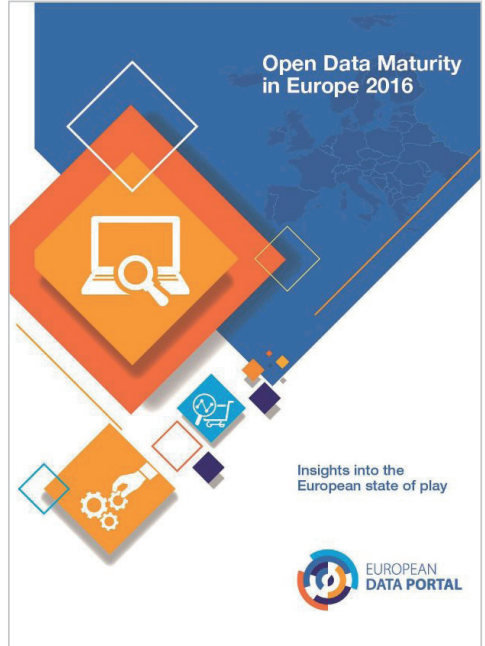

https://bit.ly/2Po5cPm 
estar siendo replicados sistemáticamente por las distintas administraciones para ganar legitimidad ante la ciudadanía y rivales políticos (García-García; Curto-Rodríguez, 2018).

Aunque a priori puede pensarse en los datos abiertos únicamente como un movimiento de regeneración democrática, sus potencialidades van mucho más allá de la transparencia. Los beneficios económicos y de tipo social (Janssen; Charalabidis; Zuiderwijk, 2012; Álvarez-García; Gértrudix-Barrio; Rajas-Fernández, 2014; Jetzek; Avital; Bjorn-Andersen, 2014; Abella; Ortiz-de-Urbina-Criado; De-Pablos-Heredero, 2017a; Berends et al., 2017) hacen que sean vistos por muchos autores como un gran activo de futuro, que confirma al "conocimiento colectivo como motor del cambio" (ÁlvarezGarcía; Gértrudix-Barrio; Rajas-Fernández, 2014, p. 674), pero para ello no sólo es suficiente crear portales de datos abiertos; su uso y adecuada gestión resultan fundamentales.

El auge de estas iniciativas y la ausencia de criterios comúnmente aceptados para evaluar estos portales justifican la pertinencia de esta investigación. Tal como se detalla en la siguiente sección, existen algunos trabajos previos que abordan desde un punto de vista teórico las finalidades y potencialidades de estas iniciativas, así como las funciones que deberían incorporar estos portales (Junta de Castilla y León, 2012; FEMP, 2017). Pocos trabajos empíricos (entre otros, Carrasco; Sobrepere, 2015; Lourenço, 2015; Medina, 2015; Chatfield; Reddick, 2017; Thorsby et al., 2017) han evaluado este tipo de portales y los análisis son mayoritariamente de tipo cualitativo o se limitan a un número reducido de portales, por lo que no existen pautas generalmente aceptadas para evaluar su implementación (Abella; Ortiz-de-Urbina-Criado; De-Pablos-Heredero, 2014; Carrasco; Sobrepere, 2015; Lourenço, 2015).

En este contexto, este trabajo tiene un doble objetivo:

- En primer lugar, proponer una metodología para analizar los portales de datos abiertos, yendo más allá del cómputo de los conjuntos y categorías de datos facilitados para incorporar otros atributos relacionados con la accesibilidad, facilidad de uso y elementos para fomentar la participación ciudadana. El éxito de los portales de datos abiertos requiere mucho más que la mera publicación de conjuntos de datos; favorecer su uso y crear oportunidades para la participación ciudadana resulta fundamental (Janssen; Charalabidis; Zuiderwijk, 2012; Carrasco; Sobrepere, 2015; Lourenço, 2015).

- En segundo lugar, aplicar ese método a una muestra de municipios españoles, para determinar si son acordes a los principios fundamentales del gobierno abierto y establecer qué áreas deberían centrar la atención en el futuro.

\section{Contextualización}

\subsection{Requisitos y beneficios de los datos abiertos}

Una vez introducido el concepto de datos abiertos, cabe formularse las siguientes cuestiones: ¿Qué requisitos han de cumplir los datos para tener tal condición? ¿Qué beneficios reportan a la sociedad y a las administraciones públicas?

Existe un amplio consenso en la bibliografía sobre qué ha de entenderse por datos abiertos. La mayor parte de autores y administraciones parten de los ocho principios siguientes, definidos en 2007 por un grupo de trabajo estadounidense: https://public.resource.org/8_principles.html

- Completos. Se deben publicar todos los datos públicos disponibles, con las únicas restricciones de derechos de autor, cuestiones de seguridad o privacidad.

- Primarios. Se deben publicar sin procesar ni agregar, ofreciendo el mayor nivel de detalle posible ${ }^{3}$.

- Actualizados. Para garantizar el valor de los datos, han de publicarse tan pronto como sea posible y actualizarse periódicamente.

- Accesibles. Se deben poner a disposición del mayor número de usuarios posible. El medio ideal para cumplir con este fin será internet.

- Procesables por máquina. Las potencialidades de los datos abiertos se multiplican exponencialmente cuando se presentan en formatos estructurados que permiten su procesamiento automatizado. Los formatos no legibles por máquina no permiten que los datos puedan interconectarse, limitando su reutilización y la generación de valor añadido (Berners-Lee, 2006) ${ }^{4}$.

- Sin discriminación. Han de ser accesibles sin necesidad de registrarse, disponer de códigos o contraseñas.

- No protegidos (sin licencia). Deben estar disponibles en formatos sobre los que ninguna entidad tenga un control exclusivo, por ejemplo utilizando csv (comma-separated values) en lugar de xls (de Microsoft Office).

- Libres. Para que puedan ser reutilizados no deben estar sujetos a derechos de autor o patentes.

En cuanto a los beneficios para los ciudadanos, la transparencia y accesibilidad a la información redunda en la igualdad de oportunidades y reducción de las discriminaciones al permitir que se erradiquen los grupos de presión y de poder 
basados en la información reservada (Garriga-Portolà, 2011). Además son un instrumento de lucha contra la corrupción, ya que posibilitan conocer el uso de los recursos públicos (Jetzek; Avital; Bjorn-Andersen, 2014; Cerrillo-Martínez, 2018). Más allá de la transparencia, las empresas, especialmente del sector TIC, disponen de mejores recursos para generar nuevos productos y servicios, fomentar la innovación y generar puestos de trabajo (Janssen; Charalabidis; Zuiderwijk, 2012; Jetzek; Avital; Bjorn-Andersen, 2014; Abella; Ortiz-de-Urbina-Criado; De-Pablos-Heredero, 2017a; Berends et al., 2017). El sector infomediario español ha tenido un crecimiento exponencial: se ha pasado de 163 empresas en 2011 a 662 a finales de 2017, dando empleo a más de 19.000 personas (Asedie, 2018).

La puesta a disposición de datos no sólo reporta ventajas para los ciudadanos y el sector empresarial, sino también a la propia Administración, lo que permite avanzar hacia una gobernanza inteligente (Cerrillo-Martínez, 2018). La apertura de datos permite a la Administración valorar y controlar su calidad y mejorar la eficiencia administrativa, por ejemplo, mediante el análisis de tendencias de la calidad, plazos de tramitación, mejora en la toma de decisiones y anticipo de situaciones, conductas o áreas de riesgo (Garriga-Portolà, 2011; Janssen; Charalabidis; Zuiderwijk, 2012; Carrara et al., 2015; Jetzek; Avital; Bjorn-Andersen, 2014; Cerrillo-Martínez, 2018). Carrara et al. (2015, pp. 11-12; p. 84) indican que con su uso se podrían ahorrar 1.700 millones de euros hasta 2020, principalmente por la puesta en común de datos y acceso más rápido a los mismos, así como reducción de atascos en carreteras, energía utilizada, o número de muertes por la mejora del tiempo de respuesta ante un accidente. Las administraciones públicas también se benefician de estas iniciativas por una cuestión de percepción ciudadana. Con una mayor transparencia, participación y colaboración ciudadana, debería mejorar la imagen que los ciudadanos tienen de las administraciones públicas (García-García, 2014; Guillamón; Ríos-Martínez; Vicente-Oliva, 2011).

\subsection{Antecedentes y estudios previos}

Los primeros portales de open data surgieron en EUA y Reino Unido. Corojan y Campos-Domínguez (2011, pp. 31-37) hacen un análisis exhaustivo de las políticas que rodean a los datos abiertos en ambos países, señalando como factores explicativos de su importancia la legislación y proyectos impulsados por la administración central (Data.gov: Empowering people en EUA y Data.gov.uk: Opening up government en Reino Unido).

La relativa novedad que suponen los proyectos de datos abiertos hace que aún no se haya investigado en profundidad sobre estos portales. La bibliografía va en aumento, pero se ha dirigido fundamentalmente a explicar finalidades y posibilidades, sin existir pautas generalmente aceptadas para evaluar su implementación (Susha et al., 2014; Carrasco; Sobrepere, 2015; Lourenço, 2015). A pesar de ser todavía escasos, existen algunos ejemplos de guías sobre el desarrollo de portales de datos abiertos, como las de la Junta de Castilla y León (2012) o la FEMP (2017), que incluyen entre otras las siguientes funciones:

- buscador de conjuntos de datos,

- ejemplos de aplicaciones desarrolladas,

- términos de uso,

- información de contacto,

- posibilidad de que sean los propios ciudadanos los que soliciten conjuntos de datos que crean útiles.

En cuanto a trabajos de tipo empírico, Carrasco y Sobrepere (2015) analizaron las iniciativas de datos abiertos en 15 municipios españoles a través de entrevistas y encuestas a los responsables de su implementación. Según concluyen, aunque hay diferencias considerables entre los municipios estudiados, los datos que se ofrecen son principalmente sobre temas no controvertidos desde el punto de vista político (como demografía o transporte) frente a otros que pueden ser una fuente de polémica para los gestores municipales (como datos tributarios, sanitarios o índices de delincuencia).

Lourenço (2015) propone una serie de características para evaluar los portales de datos abiertos para fines de transparencia y rendición de cuentas y los aplica a los portales de datos abiertos de 7 países, mediante un estudio cualitativo. Sus resultados muestran importantes carencias de tipo estructural y organizativo.

Medina (2015) analizó, también con un enfoque cualitativo, 10 iniciativas de datos abiertos a nivel municipal, 7 españolas y 3 internacionales, señalando la necesidad de mejorar el contacto y la reutilización de la información. Asimismo, también propuso mejoras en aspectos cualitativos (explicación de conceptos clave y objetivos, publicación de datos de contacto, licencias de uso, utilización de formatos con niveles altos de reutilización, integración con redes sociales y sistemas de discusión, entre otros) para que estos portales se adapten a todo tipo de usuarios.

Chatfield y Reddick (2017) Ilevaron a cabo un análisis longitudinal de tipo cualitativo de las 20 entidades locales australianas más pobladas, observando grandes variaciones en las funciones y número de conjuntos de datos divulgados. Sus resultados también revelan que la existencia de una política de datos abiertos y de un portal propio de datos abiertos favorece las mejoras de estos portales a lo largo del tiempo. La inclusión en el análisis de ciudades con portal propio y ciudades que alojan sus datos en el portal regional o estatal es una limitación importante de este trabajo, junto con el reducido número de indicadores analizados. 
Máchová y Lněnička (2017) propusieron un modelo para evaluar la calidad de los portales de datos abiertos, que aplicaron en un análisis cuantitativo de 67 portales de datos abiertos a nivel nacional, utilizando escalas de respuesta tipo Likert para la valoración de los atributos analizados, introduciendo una mayor subjetividad. Sus resultados evidencian altos grados de heterogeneidad y la necesidad de publicación de estándares de calidad para estos portales.

El trabajo de Thorsby et al. (2017) es una referencia importante para la investigación desarrollada en este trabajo, ya que elaboró un ranking de iniciativas de datos abiertos en 36 ciudades estadounidenses, a partir de una lista de atributos y ponderaciones. Los resultados muestran que los portales analizados se encuentran en etapas iniciales de desarrollo y presentan carencias en diseño, herramientas de análisis, visualización y ayuda que permitan a los usuarios comprender los datos.

De estos estudios previos, sólo Máchová y Lněnička (2017) y Thorsby et al. (2017) otorgan puntuaciones totales a los portales de datos abiertos analizados. No obstante, la metodología que plantean tiene algunas limitaciones. Por ejemplo, Máchová y Lněnička (2017) no valoran el número de conjuntos de datos publicados sino que definen el siguiente ítem: "El portal indica el número de datos que incluye", por lo que se valora si se indica el número de conjuntos de datos divulgados, con independencia del valor que tome este indicador. Con este ejemplo también se aprecia que la valoración con una escala Likert (1=totalmente en desacuerdo, 2=en desacuerdo, 3=neutro, 4=de acuerdo, 5=totalmente de acuerdo) carece de sentido para este ítem, ya que sólo dos de las opciones son posibles (o se indica o no se indica el número de conjuntos de datos). Además, un portal que no cumpliese con ninguno de los items propuestos obtendría una puntuación de 28 (el número de items analizados).

Por su parte, Thorsby et al. (2017) definen puntuaciones variables para cada ítem, que en algunos casos no resultan apropiadas para el análisis de portales municipales en España. Por ejemplo, un ayuntamiento como máximo puede obtener 5 puntos en función del número de conjuntos de datos alojados, siempre que disponga de más de 100 conjuntos de datos por cada 100.000 habitantes, lo cual es una cifra bastante elevada. Madrid necesitaría 3.174 conjuntos de datos para obtener 5 puntos y, tal como se observa posteriormente en los resultados de nuestro trabajo empírico (tabla 1), el máximo de conjuntos de datos de nuestra muestra es 766. Otros de los items que analizan en nuestra opinión tienen una menor importancia, pero también se valoran con 5 o más puntos. Por ejemplo, el hecho de que los datos estén organizados por categorías se valora con dos items y una puntuación que puede llegar hasta los 10 puntos y la existencia de dirección de contacto o datos en formatos legibles por máquina se valora con 20 puntos cada uno. Por ello estos trabajos previos se han tomado como punto de partida para definir los items a analizar, pero se consideró necesario definir un nuevo método de análisis, tal como se ilustra en la siguiente sección.

El éxito de los portales de datos abiertos
requiere mucho más que la mera publi-
cación de conjuntos de datos: favorecer
su uso y crear oportunidades para la
participación ciudadana es fundamental

\section{Metodología}

La investigación tiene como objeto de estudio los portales de datos abiertos de municipios españoles. Para ello, se utiliza un conjunto de items y se elaboran tablas de frecuencias y un ranking de dichos portales. Además se analiza la información económico-financiera que ofrecen los municipios en sus portales de datos abiertos, por ser fundamental para la rendición de cuentas, la lucha contra la corrupción y la evaluación de la sostenibilidad y eficacia de la prestación de servicios públicos (Pina; Torres; Royo, 2010). Máchová y Lněnička (2017, p. 34) destacaron que es necesario divulgar más información de tipo económico-financiero en los portales de datos abiertos para incrementar los niveles de transparencia y rendición de cuentas. Además, según Berends et al. (2017), las categorías de "administración y sector público" y "economía y finanzas" son las dos más demandadas por las empresas que utilizan datos abiertos.

La muestra la forman las capitales de provincia y el resto de ciudades españolas de más de 100.000 habitantes. El objeto de análisis no son las webs o portales de transparencia genéricos del municipio, sino los de datos abiertos. La casuística es realmente variada, con ciudades que tienen una web específica de datos abiertos, mientras que algunos municipios incluyen un apartado de datos abiertos dentro de sus páginas de transparencia o gobierno abierto, en cuyo caso el análisis se refiere a dicha sección.

En diciembre de 2017 se realizó una búsqueda exhaustiva en las webs de los municipios de la muestra. Del total de 52 capitales de provincia españolas -incluyendo las ciudades autónomas de Ceuta y Melilla-tan sólo 22 contaban con un portal de 
datos abiertos y entre las 27 ciudades que superan los 100.000 habitantes sin ser capital de provincia, el número era de 10. Aunque la ciudad de Leganés sí contaba con un portal de datos abiertos, se trató como si no lo tuviera ya que no había sido actualizado desde 2015. Por tanto, se han analizado 32 portales (el listado de ciudades aparece en la tabla

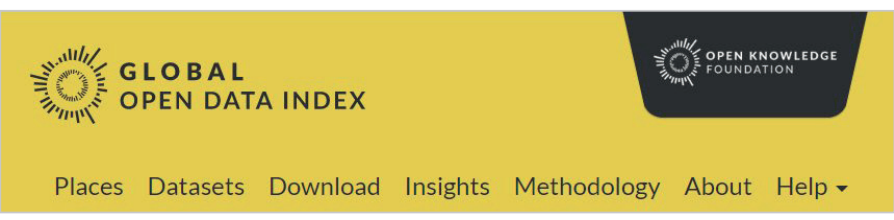

https://index.okfn.org/methodology 7), lo que supone que sólo un $40 \%$ de los municipios de la muestra cuentan con un portal de datos abiertos.

Para analizar los portales se elaboró una lista de 28 items, tomando como referencia las investigaciones previas españolas e internacionales, la norma UNE 178301 de Aenor (2015), el Global Open Data Index y la propia evidencia obtenida en el análisis realizado (el Anexo detalla los items analizados, los criterios de valoración y referencias a trabajos previos que han utilizado los items propuestos).

Los items se han agrupado en cuatro dimensiones:

- Catálogo de datos (6 items)

- Contenido del portal (10)

- Accesibilidad y visualización (7)

- Participación ciudadana (5)

En general, cada ítem se valora con 1 punto en caso de estar presente en el portal o 0 en caso contrario, excepto tres de la primera dimensión (véase el Anexo), que permiten una puntuación mayor a 1 en función del número de conjuntos de datos y categorías del portal, así como de la publicación de datos en formatos legibles por máquina utilizando los estándares abiertos definidos por el World Wide Web Consortium, como RDF, es decir, que alcanzan la categoría de 4 estrellas de Berners-Lee (2006) ${ }^{5}$.

La primera dimensión "Catálogo de datos" hace referencia a los datos ofrecidos: número de conjuntos de datos, categorías, formatos legibles por máquina o explicación de los metadatos utilizados y tiene el mayor peso en la puntuación total (representa casi el 60\% de la puntuación final, 31 puntos de los 53 posibles, según se detalla en el Anexo, por considerar que los datos en sí son el principal elemento que deben contener estos portales).

La dimensión "Contenido del portal" analiza la presencia de otras aplicaciones o contenidos, más allá de los propios datos. Según Carrasco y Sobrepere (2015, p. 634) y Lourenço (2015), abrir conjuntos de datos no es suficiente; también es necesario considerar el nivel de interacción y su utilización. Algunos de los items analizados son específicos para desarrolladores o personas especializadas en informática (presencia de APIs), pero la mayoría son útiles para todo tipo de público, como la política de datos abiertos de la institución o la licencia para la reutilización de datos.

La dimensión "Accesibilidad y visualización" hace referencia al diseño y facilidad de uso de los portales, que tampoco han de descuidarse si se pretende una interacción real con los ciudadanos. Se incluye la existencia de una sección de ayuda, buscador, posibilidad de filtrar u ordenar los conjuntos de datos, que se puedan manipular sin necesidad de descarga o posibilidad de crear gráficos online.

La última dimensión, "Participación ciudadana", valora aspectos como la posibilidad de que los usuarios sugieran conjuntos de datos, los valoren, den sus opiniones, etc. Según Janssen, Charalabidisy Zuiderwijk (2012), no sólo deben publicarse datos, sino buscar de forma activa las opiniones de los usuarios y utilizar esa información para mejorar la Administración.

Para analizar los datos recopilados, en primer lugar se han realizado análisis descriptivos mediante tablas de frecuencias, máximos, mínimos, medias, etc. Para cuantificar los niveles de desarrollo de los portales de datos abiertos se han calculado puntuaciones, tanto a nivel global como para cada una de las cuatro dimensiones analizadas, con valores que pueden oscilar entre el 0 y el $100 \%$. Cada una de estas puntuaciones se ha calculado como la suma de las puntuaciones de los items incluidos en cada dimensión y dividiendo por la máxima puntuación posible, según se desprende de los criterios de codificación establecidos en el Anexo. Por ejemplo, para la puntuación total, la suma de la puntuación de todos los items individuales se ha divido entre 53, multiplicando el resultado final por 100 para obtener porcentajes de divulgación. La puntuación total permite elaborar un ranking de los portales analizados.

\begin{abstract}
Se analizan los portales de datos abiertos utilizando 28 items agrupados en cuatro dimensiones: catálogo de datos, contenido del portal, accesibilidad y visualización, y participación ciudadana
\end{abstract}

\section{Análisis de resultados}

\subsection{Análisis descriptivo de los items analizados}

En primer lugar, se presenta el análisis descriptivo de los dos atributos de tipo cuantitativo de la dimensión "Catálogo de datos" (conjuntos datos y número de categorías, véase la tabla 1). A continuación, mediante análisis de frecuencias, se presentan las tendencias más destacadas que han sido observadas en el resto de items de tipo dicotómico (tablas 2-5). 
La media de conjuntos de datos alojados en los portales es de 141,5 mientras que la mediana se sitúa en 85,5 , una cifra considerablemente inferior. Esto sugiere la existencia de datos atípicos: son unas pocas ciudades las que alojan un conjunto de datos mucho mayor que el resto. El valor de la desviación típica, mínimo y máximo corrobora la gran dispersión existente en el número de conjuntos de datos publicados. En relación con el número de categorías, la media es de 11 , aunque también se observan variaciones importantes.

La tabla 2 muestra la frecuencia del resto de items analizados en la dimensión "Catálogo de datos". La gran mayoría de portales tienen datos en formato legible por máquina (91\%) y muestran la fecha de última actualización (81\%). No obstante, sólo el $28 \%$ de los portales contienen metadatos que expliquen el contenido de los conjuntos de datos, aspecto fundamental para programadores y desarrolladores y facilitar su reutilización. Sólo el $38 \%$ de los portales informan del número de descargas de cada conjunto de datos.

En la dimensión "Contenido del portal" (tabla 3), un 81\% incluye la licencia para la utilización de datos, requisito obligatorio por ley. El porcentaje disminuye bastante cuando se consideran aspectos como si contienen APIs (47\%), cualidad dirigida a un público más experto, o la existencia de una lista de preguntas frecuentes (9\%), tutoriales (3\%) o demostradores (13\%), las cuales parecen más útiles para usuarios no expertos. Estos resultados muestran un cierto olvido de los usuarios no especializados. Además, sólo el $9 \%$ de los portales publican estadísticas de uso de los datos que alojan, lo que confirma las conclusiones obtenidas por Carrasco y Sobrepere (2015, p. 637) en una muestra más reducida de municipios españoles: en general, no se recogen ni analizan estadísticas sobre la utilización de los datos. Tan sólo 2 las de 32 ciudades (Valencia y Barcelona) indican que cuentan con algún tipo de certificado, en concreto el ISO 37120 (desarrollo sostenible de comunidades, indicadores de servicios locales y calidad de vida).

En relación con la "Accesibilidad y visualización" (tabla 4), el 91\% de los portales estructuran en categorías los conjuntos de datos y en el $78 \%$ hay un buscador de conjuntos de datos, aunque sólo algo más de la mitad (53\%) permite ordenar los datos en función de otros criterios. Sólo un tercio ofrece la posibilidad de manipular los datos online y todavía menos común es la posibilidad de crear gráficos online (13\%). Además, sólo el $9 \%$ tiene una sección propia de ayuda.

Ninguno de los items de la dimensión "Participación ciudadana" (tabla 5) está presente en más de la mitad de los portales analizados. El $47 \%$ permite al ciudadano solicitar nuevos conjuntos de datos y el $41 \%$ permite aportar ideas sobre cómo mejorar el repositorio de datos. Ningún portal cuenta con información completa de contacto (persona responsable, número de teléfono y correo electrónico) y muy pocos cuentan con concursos o actividades para promover su utilización.

A modo de resumen, la mayoría de portales parecen ir dirigidos a un público especializado. Las cualidades dirigidas a públicos expertos (APIs o formatos legibles por máquina) parecen ser más comunes que las herramientas de ayuda o guías para el usuario de los portales. Esta propensión fue advertida también por Thorsby et al. (2017, p. 59), constatando que la mayoría de iniciativas en ciudades estadounidenses iban dirigidas a liberar datos para construir aplicaciones. No obstante, en la muestra analizada sólo la mitad de los portales contiene un repositorio de las aplicaciones creadas con los conjuntos de datos ofrecidos, por lo que resulta necesario fomentar su difusión.
Tabla 1. Conjuntos y categorías de datos

\begin{tabular}{|l|c|c|}
\cline { 2 - 3 } \multicolumn{1}{c|}{} & $\begin{array}{c}\text { Conjuntos de } \\
\text { datos }\end{array}$ & $\begin{array}{c}\text { Número de } \\
\text { categorías }\end{array}$ \\
\hline Media & 141,5 & 11,2 \\
\hline Mediana & 85,5 & 11 \\
\hline Moda & 37 & 19 \\
\hline Desviación típica & 183,1 & 6,9 \\
\hline Mínimo & 2 & 0 \\
\hline Máximo & 766 & 22 \\
\hline
\end{tabular}

Tabla 2. Resto de items del Catálogo de datos

\begin{tabular}{|l|l|}
\hline \multicolumn{1}{|c|}{ Ítem } & $\%$ \\
\hline Formato legible por máquina & 91 \\
\hline Metadatos & 28 \\
\hline Fecha de la última actualización de los datos & 81 \\
\hline Número de descargas de cada conjunto de datos & 38 \\
\hline
\end{tabular}

Tabla 3. Contenido del portal

\begin{tabular}{|l|c|}
\hline \multicolumn{1}{|c|}{ Ítem } & $\%$ \\
\hline Apartado de novedades & 22 \\
\hline Demostrador & 13 \\
\hline Lista de preguntas frecuentes & 9 \\
\hline Tutoriales & 3 \\
\hline Ejemplos de aplicaciones desarrolladas & 50 \\
\hline APIs & 47 \\
\hline Estadísticas de uso & 9 \\
\hline Política de datos abiertos & 75 \\
\hline Licencia para la utilización de datos & 81 \\
\hline Certificados & 6 \\
\hline
\end{tabular}

Tabla 4. Accesibilidad y visualización

\begin{tabular}{|l|c|}
\hline \multicolumn{1}{|c|}{ Ítem } & $\%$ \\
\hline Sección de ayuda & 9 \\
\hline Crear gráficos online & 13 \\
\hline Manipular datos online & 34 \\
\hline Organización por categorías & 91 \\
\hline Ordenar los datos según otros criterios & 53 \\
\hline $\begin{array}{l}\text { Datos clasificados por estrellas según el grado de } \\
\text { apertura }\end{array}$ & 16 \\
\hline Buscador & 78 \\
\hline
\end{tabular}

Tabla 5. Participación ciudadana

\begin{tabular}{|l|c|}
\hline \multicolumn{1}{|c|}{ Ítem } & $\%$ \\
\hline Sugerir nuevos conjuntos de datos & 47 \\
\hline Comentarios o ideas de cómo mejorar el servicio & 41 \\
\hline Valoración del conjunto de datos & 19 \\
\hline Información completa de contacto & 0 \\
\hline Concursos o actividades para promover el uso & 6 \\
\hline
\end{tabular}




\subsection{Puntuaciones de las dimensiones estudiadas}

Como puede observarse en la tabla 6, la dimensión que tiene una media mayor es "Catálogo de datos" (57,9\%), presentando el resto de dimensiones puntuaciones menores al 50\%, si bien existen grandes variaciones en las puntuaciones en todas las dimensiones. En "Participación ciudadana" la puntuación media es 22,5\%, siendo la moda el $0 \%$, lo cual indica que la mayor parte de portales tiene deficiencias importantes en materia de interacción. La puntuación total media es del $47,5 \%$. Tal como se aprecia en la tabla 7, hay mucha variedad en las puntuaciones bajas, pero la mayor parte de las puntuaciones altas se sitúa entre el 50 y el $70 \%$, con sólo 3 ciudades con una puntuación mayor.

Tabla 6. Puntuaciones de las dimensiones analizadas

\begin{tabular}{|l|c|c|c|c|}
\cline { 2 - 5 } & $\begin{array}{c}\text { Catálogo de datos } \\
\%\end{array}$ & $\begin{array}{c}\text { Contenido } \\
\%\end{array}$ & $\begin{array}{c}\text { Accesibilidad y } \\
\text { visualización } \\
\%\end{array}$ & $\begin{array}{c}\text { Participación } \\
\text { ciudadana } \\
\%\end{array}$ \\
\hline Media & 57,9 & 31,6 & 42,0 & 22,5 \\
\hline Mediana & 60,5 & 30,0 & 42,9 & 20,0 \\
\hline Moda & 96,8 & 30,0 & 42,9 & 0 \\
\hline Desviación típica & 28,6 & 19,4 & 21,1 & 24,8 \\
\hline Mínimo & 3,9 & 0 & 0 & 22,1 \\
\hline Máximo & 100 & 80,0 & 85,7 & 0 \\
\hline
\end{tabular}

\subsection{Ranking de municipios}

Las ciudades mejor situadas en el ranking (tabla 7) son Madrid y Barcelona, las dos más pobladas del país, con puntuaciones por encima del $80 \%$. Si a ello le añadimos el hecho de que Valencia, Sevilla, Zaragoza y Málaga, las cuatro siguientes en población, están dentro del top 10, los resultados parecen sugerir que las ciudades más pobladas suelen tener un portal de datos abiertos más desarrollado. De hecho, el coeficiente de correlación de Pearson entre la población de la ciudad y la puntuación total es de 0,63 (significativo al 1\%), lo cual confirma, tal como han obtenido Thorsby et al. (2017) para ciudades de EUA, que el tamaño de la ciudad, y por ende el nivel de recursos, es un factor crítico a la hora de explicar el nivel de desarrollo de estas iniciativas.

Tabla 7. Ranking de portales de datos abiertos

\begin{tabular}{|c|c|c|c|c|c|c|}
\hline & Ciudad & $\begin{array}{c}\text { Catálogo de } \\
\text { datos } \\
\%\end{array}$ & $\begin{array}{c}\text { Contenido } \\
\%\end{array}$ & $\begin{array}{c}\text { Accesibilidad y } \\
\text { visualización } \\
\%\end{array}$ & $\begin{array}{c}\text { Participación } \\
\text { ciudadana } \\
\%\end{array}$ & $\begin{array}{c}\text { Total } \\
\%\end{array}$ \\
\hline 1 & Barcelona & 96,8 & 80 & 71,4 & 40 & 84,9 \\
\hline 2 & Madrid & 100 & 50 & 57,1 & 80 & 83,0 \\
\hline 3 & Málaga & 96,8 & 30 & 71,4 & 0 & 71,7 \\
\hline 4 & Valencia & 73,5 & 70 & 57,1 & 60 & 69,4 \\
\hline 5 & Terrassa & 84,8 & 40 & 71,4 & 20 & 68,5 \\
\hline 6 & Alcobendas & 87,1 & 40 & 57,1 & 20 & 67,9 \\
\hline 7 & Sevilla & 79,7 & 40 & 57,1 & 60 & 67,4 \\
\hline 8 & Bilbao & 89,4 & 30 & 42,9 & 40 & 67,4 \\
\hline 9 & Gijón & 93,5 & 30 & 42,9 & 0 & 66,0 \\
\hline 10 & Zaragoza & 75,5 & 60 & 42,9 & 40 & 64,9 \\
\hline 11 & Santander & 63,9 & 50 & 57,1 & 60 & 60,0 \\
\hline 12 & Las Palmas de Gran Canaria & 61,9 & 50 & 57,1 & 60 & 58,9 \\
\hline 13 & Pamplona & 90,3 & 0 & 28,6 & 20 & 58,5 \\
\hline 14 & Vitoria & 68,4 & 40 & 28,6 & 60 & 57,0 \\
\hline 15 & Valladolid & 84,5 & 20 & 14,3 & 20 & 57,0 \\
\hline 16 & I'Hospitalet de Llobregat & 48,7 & 60 & 85,7 & 40 & 54,9 \\
\hline 17 & Sabadell & 74,2 & 30 & 28,6 & 20 & 54,7 \\
\hline 18 & Cáceres & 59,0 & 30 & 57,1 & 20 & 49,6 \\
\hline 19 & Santa Cruz de Tenerife & 54,2 & 30 & 42,9 & 20 & 44,9 \\
\hline 20 & Lleida & 47,1 & 30 & 28,6 & 0 & 37,0 \\
\hline 21 & Badalona & 43,2 & 20 & 42,9 & 0 & 34,7 \\
\hline 22 & Girona & 46,1 & 10 & 42,9 & 0 & 34,5 \\
\hline
\end{tabular}




\begin{tabular}{|c|l|c|c|c|c|}
\hline 23 & Mataró & 37,7 & 20 & 42,9 & 0 \\
\hline 24 & Santa Coloma de Gramenet & 37,7 & 20 & 42,9 & 0 \\
\hline 25 & Reus & 23,2 & 30 & 42,9 & 40 \\
\hline 26 & Cuenca & 33,9 & 20 & 28,6 & 0 \\
\hline 27 & San Cristóbal de la Laguna & 20,6 & 20 & 57,1 & 0 \\
\hline 28 & Oviedo & 29,0 & 20 & 14,3 & 0 \\
\hline 29 & Albacete & 15,2 & 10 & 14,3 & 0 \\
\hline 30 & Lugo & 16,1 & 0 & 14,3 & 0 \\
\hline 31 & San Sebastián & 18,1 & 0 & 0 & 0 \\
\hline 32 & Palma de Mallorca & 3,9 & 30 & 0 & 1,3 \\
\hline
\end{tabular}

\subsection{Información económico-financiera}

Más de la mitad de los ayuntamientos publica el presupuesto aprobado (66\%), aunque sólo un $44 \%$ divulga información sobre el estado de liquidación del presupuesto y muy pocos (13\%) sobre las modificaciones presupuestarias (véase la tabla 8). La información económico-financiera ofrecida a los ciudadanos es incompleta: de nada sirve publicar el presupuesto aprobado si no se informa sobre su grado de ejecución o variaciones posteriores, aspectos que reflejan verdaderamente en qué se gastan los recursos públicos.

Tabla 8. Información económico-financiera

\begin{tabular}{|l|c|}
\hline \multicolumn{1}{|c|}{ Ítem } & $\%$ \\
\hline Presupuesto aprobado & 66 \\
\hline Modificaciones presupuestarias & 13 \\
\hline Estado de liquidación del presupuesto & 44 \\
\hline Cuentas anuales o niveles de endeudamiento & 25 \\
\hline Contratos celebrados / Perfil del contratante & 56 \\
\hline Subvenciones concedidas & 44 \\
\hline
\end{tabular}

Tan sólo uno de cada cuatro ayuntamientos incluye datos referidos a las cuentas anuales o niveles de endeudamiento, indispensables para evaluar la capacidad de la entidad de hacer frente a sus obligaciones y prestar servicios a largo plazo. Esta información puede ser decisiva para los ciudadanos a la hora de ejercer sus derechos políticos, ya que ayuda a valorar la gestión realizada (Alcalde-Hernández, 2012).

\section{Discusión y conclusiones}

Los portales de datos abiertos son buques insignia de las iniciativas de gobierno abierto, lo que justifica el gran auge que han tenido recientemente. No obstante, el número de trabajos que ha propuesto indicadores para evaluar el nivel de desarrollo de estos portales es todavía reducido y los trabajos previos que proponen una metodología para su evaluación presentan limitaciones, por lo que una primera contribución de este trabajo es el método propuesto para la evaluación de los portales de datos abiertos de los municipios. Este trabajo no ha tenido como objetivo el análisis en profundidad del tipo de datos alojados en estos portales, sino el estudio de aspectos estructurales que se considera que deberían cumplir. Obviamente, los criterios analizados y puntuaciones otorgadas no son los únicos posibles y, aunque para su confección se han tenido en cuenta los principales estudios y guías sobre esta materia, existe cierta subjetividad. A esta limitación se le suma otra de tipo metodológico. La casuística de los portales analizados es muy variada, lo que ha dotado al proceso de obtención de datos de cierta dificultad.

A pesar del auge de las iniciativas de datos abiertos, llama la atención el bajo porcentaje de ciudades analizadas que cuenta con un portal de este tipo (40\%), lo que ha reducido a 32 el número de portales analizados. Los resultados obtenidos muestran grandes diferencias entre portales, tal como han encontrado estudios previos en otros contextos (Chatfield; Reddick, 2017; Máchová; Lněnička, 2017; Thorsby et al., 2017; Cecconi; Radu, 2018). Si bien Barcelona y Madrid obtienen puntuaciones totales superiores al $80 \%$, la media de la muestra analizada no alcanza el 50\%, incluso a pesar de que la dimensión con más peso ("Catálogo de datos") es la que obtiene mayores puntuaciones medias. Este hecho es indicativo de una tendencia que ya advirtió Lourenço (2015): en términos generales, los portales parecen funcionar como simples repositorios de datos, obviando otros aspectos encaminados a promover la rendición de cuentas y el uso por parte de los ciudadanos.

La gran variabilidad y baja puntuación media obtenida contrasta con el hecho de que el 75\% de las ciudades analizadas cuentan con una política de datos abiertos que publican en su portal. En un estudio de entidades locales australianas (Chatfield; Reddick, 2017) sólo el 35\% de las ciudades contaba con una política de este tipo, por lo que se recomendaba fomentar su adopción (en el caso de las ciudades estadounidenses, este porcentaje ascendía al 50\% (Thorsby et al., 2017). Futuros trabajos podrían analizar el contenido de estas políticas para determinar si su enfoque -por ejemplo, interno versus externo (Chatfield; Reddick, 2017)- ayuda a explicar el grado de desarrollo de los portales de datos abiertos. Además, futuros trabajos podrían analizar qué otros factores contribuyen al desarrollo de estos portales.

Sólo un $40 \%$ de las ciudades analizadas tienen un portal de datos abiertos, y la mayoría de portales están dirigidos a un público especializado 
Nuestros resultados sugieren que el principal objetivo de los ayuntamientos españoles con la adopción de estos portales es fomentar la reutilización de datos por parte del sector privado (aunque a un nivel muy básico, ya que menos de un $30 \%$ de los portales definen los metadatos utilizados y menos del $50 \%$ permite la descarga de datos a través de APIs) más que su utilización como medio de rendición de cuentas y participación ciudadana. El análisis de la información económico-financiera puesta a disposición a través de estos portales también apunta a la falta de interés por la rendición de cuentas, ya que la información de ese tipo es en muchos casos incompleta.

En definitiva, a los municipios españoles todavía les queda mucho por avanzar en materia de datos abiertos, tanto en la adopción de este tipo de portales, como en el desarrollo de los ya existentes, especialmente en aspectos que fomenten la utilización de los datos por parte de un público no experto (como tutoriales, secciones de ayuda o demostradores), la rendición de cuentas, la participación ciudadana, y la calidad de los datos suministrados (por ejemplo, definición de los metadatos utilizados). El gobierno abierto se sustenta en el diálogo entre la Administración y los ciudadanos, por lo que las iniciativas enmarcadas en esta filosofía no deberían descuidar las demandas e intereses de los ciudadanos. Las carencias expuestas también apuntan a la necesidad de publicación y mayor difusión de guías y estándares para la puesta en práctica de este tipo de iniciativas. Además, dadas las diferencias existentes entre portales, ello podría contribuir a su homogeneidad, facilitando su utilización y la comparación de datos.

\section{Notas}

1. Aunque algunos autores sitúan las primeras manifestaciones del gobierno abierto en el espacio político británico de finales de los años 70 del siglo XX (Medina, 2015) o incluso en la Dinamarca del siglo XVI (Sandoval, 2017), la mayor parte de la bibliografía sitúa en el inicio de la Administración Obama la puesta en práctica del open government.

2. Para una discusión detallada del concepto portal de datos abiertos, véase Chatfield y Reddick (2017) y Máchová y Lněnička (2017).

3. Siguiendo este criterio y las indicaciones de la Junta de Castilla y León (2012, p. 8), se excluirán del estudio informes publicados en los portales de datos abiertos, ya que no publican los datos originales y, por tanto, no cumplen este criterio.

4. Este autor, creador de la Web, propuso una clasificación por estrellas que es un gran referente en todo lo relacionado con los datos abiertos y su legibilidad por máquinas.

5. Al igual que Chatfield y Reddick (2017), la categoría de 5 estrellas (que añade que los datos estén vinculados a los de otras organizaciones u usuarios) no se tuvo en cuenta en esta investigación por la dificultad para diferenciarla de la categoría 4 (ambas están referidas a la capacidad de reutilización de los datos). Además, los resultados de Abella, Ortiz-de-Urbina-Criado y De-Pablos-Heredero (2018), indican que ninguno de los conjuntos de datos del portal de Barcelona alcanzaba esta categoría en octubre de 2017. Sí que se añadió como ítem adicional la existencia de metadatos sobre los propios datos, tal como sugieren Berners-Lee (2006) o Abella, Ortiz-de-Urbina-Criado y De-Pablos-Heredero (2018), entre otros. La razón para atribuir 5 puntos al ítem que mide la presencia de formatos legibles por máquina es su transcendencia, ya que la mayor parte de autores (Abella; Ortiz-de-Urbina-Criado; De-Pablos-Heredero, 2014, p. 584; Chatfield y Reddick 2017, p. 232; Máchová y Lněnička, 2017, p. 22) incluyen este requisito en su definición de portales de datos abiertos. Carrara, Nieuwenhuis y Vollers (2016) y Thorsby et al. (2017) también asignaron una mayor puntuación a este ítem.

\section{Referencias}

Abella, Alberto; Ortiz-de-Urbina-Criado, Marta; De-Pablos-Heredero, Carmen (2014). "Meloda, métrica para evaluar la reutilización de datos abiertos". El profesional de la información, v. 23, n. 6, pp. 582-588.

https://doi.org/10.3145/epi.2014.nov.04

Abella, Alberto; Ortiz-de-Urbina-Criado, Marta; De-Pablos-Heredero, Carmen (2017a). "A model for the analysis of data-driven innovation and value generation in smart cities' ecosystems". Cities, v. 64, pp. 47-53.

https://doi.org/10.1016/j.cities.2017.01.011

Abella, Alberto; Ortiz-de-Urbina-Criado, Marta; De-Pablos-Heredero, Carmen (2017b). La reutilización de datos abiertos: una oportunidad para España. Fundación Cotec para la Innovación.

https://cotec.es/proyecto/reutilizacion-de-datos-abiertos-una-oportunidad-para-espana

Abella, Alberto; Ortiz-de-Urbina-Criado, Marta; De-Pablos-Heredero, Carmen (2018). "Indicadores de calidad de los datos abiertos: el caso del portal de datos abiertos de Barcelona". El profesional de la información, v. 27, n. 2, pp. 375-382. https://doi.org/10.3145/epi.2018.mar.16

Aenor (2015). Ciudades inteligentes. Datos abiertos (open data). Norma UNE 178301. Asociación Española de Normalización y Certificación (Aenor). 
Alcalde-Hernández, José-Carlos (2012). “Transparencia y acceso a la información económico-financiera del sector público". Revista española de control externo, v. 14, n. 42, pp. 17-40.

https://bit.ly/36yEVU7

Álvarez-García, Sergio; Gértrudix-Barrio, Manuel; Rajas-Fernández, Mario (2014). "La construcción colaborativa de bancos de datos abiertos como instrumento de empoderamiento ciudadano". Revista latina de comunicación social, n. 69, pp. 661-683.

https://doi.org/10.4185/rlcs-2014-1029

Asedie (2018). VI Informe Asedie del sector infomediario. Asociación Multisectorial de la Información.

http://www.asedie.es/assets/informe-sector-infomediario-2018.pdf

Berends, Jorn; Carrara, Wendy; Engbers, Wander; Vollers, Heleen (2017). Reusing open data. A study on companies transforming open data into economic \& societal value. European Commission. Directorate General for Communications Networks, Content and Technology.

https://www.europeandataportal.eu/sites/default/files/re-using_open_data.pdf

Berners-Lee, Tim (2006). Linked data.

https://www.w3.org/Designlssues/LinkedData.htm/

Carrara, Wendy; Nieuwenhuis, Margriet; Vollers, Heleen (2016). Open data maturity in Europe 2016. Insights into the European state of play. European Commission. Directorate General for Communications Networks, Content and Technology.

https://www.europeandataportal.eu/sites/default/files/edp_landscaping_insight_report_n2_2016.pdf

Carrara, Wendy; San-Chan, Wae; Fischer, Sandra; Van-Steenbergen, Eva (2015). Creating value through open data: $A$ study on the impact of re-use of public data resources. European Commission. Directorate General for Communications Networks, Content and Technology.

https://www.europeandataportal.eu/sites/default/files/edp_creating_value_through_open_data_0.pdf

Carrasco, Carlos; Sobrepere, Xavier (2015). “Open government data: An assessment of the Spanish municipal situation”. Social science computer review, v. 33, n. 5, pp. 631-644.

https://doi.org/10.1177/0894439314560678

Cecconi, Gianfranco; Radu, Cosmina (2018). Open data maturity in Europe 2018. European Commission. Directorate General for Communications Networks, Content and Technology.

https://www.europeandataportal.eu/sites/default/files/edp_landscaping_insight_report_n4_2018.pdf

Cerrillo-Martínez, Agustí (2018). “Datos masivos y datos abiertos para una gobernanza inteligente”. El profesional de la información, v. 27, n. 5, pp. 1128-1135.

https://doi.org/10.3145/epi.2018.sep.16

Chatfield, Akemi-Takeoka; Reddick, Christopher G. (2017). “A longitudinal cross-sector analysis of open data portal service capability: The case of Australian local governments". Government information quarterly, v. 34, n. 2, pp. $231-243$. https://doi.org/10.1016/j.giq.2017.02.004

Corojan, Ana; Campos-Domínguez, Eva (2011). Gobierno abierto: alcance e implicaciones. Madrid: Fundación Ideas. ISBN: 9788415018735

https://dialnet.unirioja.es/descarga/libro/572551.pdf

Criado, José-Ignacio; Ruvalcaba-Gómez, Edgar (2016). ¿Qué es y qué se entiende por gobierno abierto? Análisis de la percepción e implementación del gobierno abierto en el ámbito local español.

https://novagob.org/wp-content/uploads/2017/05/NovagobAcademia_2016_1_GobiernoAbierto-AALL.pdf

Curto-Rodríguez, Ricardo (2015). “Los portales de datos autonómicos y la rendición de cuentas”. Auditoría pública, n. 66, pp. 75-83.

https://bit.ly/3813Zts

FEMP (2017). Datos abiertos. Guía estratégica para su puesta en marcha. Conjunto de datos mínimos a publicar. Red de Entidades Locales por la Transparencia y la Participación Ciudadana.

http://femp.femp.es/files/3580-1617-fichero/Guía Datos Abiertos.pdf

Ferrer-Sapena, Antonia; Peset, Fernanda; Aleixandre-Benavent, Rafael (2011). “Acceso a los datos públicos y su reutilización: open data y open government". El profesional de la información, v. 20, n. 3, pp. 260-269.

https://doi.org/10.3145/epi.2011.may.03

García-García, Jesús (2014). “Gobierno abierto: transparencia, participación y colaboración en las administraciones públicas". Innovar, v. 24, n. 54, pp. 75-88.

https://doi.org/10.15446/innovar.v24n54.46441 
García-García, Jesús; Curto-Rodríguez, Ricardo (2018). “Divulgación de información pública de las comunidades autónomas españolas (2013-2017): portal de datos abiertos, portal de transparencia y web institucional". El profesional de la información, v. 27, n. 5, pp. 1051-1060.

https://doi.org/10.3145/epi.2018.sep.09

Garriga-Portolà, Marc (2011). “¿Datos abiertos? Sí, pero de forma sostenible”. El profesional de la información, v. 20, n. 3, pp. 298-303.

https://doi.org/10.3145/epi.2011.may.08

Guillamón, María-Dolores; Ríos-Martínez, Ana-María; Vicente-Oliva, Cristina (2011). “Transparencia financiera de los municipios españoles. Utilidad y factores relacionados". Auditoría pública, n. 55, pp. 109-116.

https://bit.ly/2rE9id6

Janssen, Marijn; Charalabidis, Yannis; Zuiderwijk, Anneke (2012). "Benefits, adoption barriers and myths of open data and open government". Information systems management, v. 29, n. 4, pp. 258-268.

Jetzek, Thorhildur; Avital, Michel; Bjorn-Andersen, Niels (2014). “Data-driven innovation through open government data". Journal of theoretical and applied electronic commerce research, v. 9, n. 2, pp. 100-120.

https://scielo.conicyt.cl/scielo.php?script=sci_arttext\&pid=S0718-18762014000200008

Junta de Castilla y León (2012). Open data para administraciones locales. Valladolid: Observatorio Regional de la Sociedad de la Información.

http://bibliotecadigital.jcyl.es/es/consulta/registro.cmd?id=18538

Lourenço, Rui-Pedro (2015). "An analysis of open government portals: A perspective of transparency". Government information quarterly, v. 32, n. 3, pp. 323-332.

https://doi.org/10.1016/j.giq.2015.05.006

Máchová, Renata; Lněnička, Martin (2017). "Evaluating the quality of open data portals on the national level”. Journal of theoretical and applied electronic commerce research, v. 12, n. 21, pp. 21-41.

https://scielo.conicyt.cl/scielo.php?script=sci_abstract\&pid=S0718-18762017000100003

Marcos-Martín, Carlos; Soriano-Maldonado, Salvador-Luis (2011). “Reutilización de la información del sector público y open data en el contexto español y europeo. Proyecto Aporta". El profesional de la información, v. 20, n. 3, pp. $291-297$. https://ddoi.org/10.3145/epi.2011.may.07

Medina, Vanessa (2015). "Análisis de proyectos 'open data' en España: propuestas de mejora”. Cuadernos de gestión de información, v. 5, n. 1, pp. 53-72.

https://revistas.um.es/gesinfo/article/view/232231

Obama, Barack (2009). Memorandum for the heads of executive departments and agencies. Executive Office of the President of the United States.

https://www.whitehouse.gov/sites/whitehouse.gov/files/omb/memoranda/2009/m09-12.pdf

Pina, Vicente; Torres, Lourdes; Royo, Sonia (2010), "Is e-government promoting convergence towards more accountable local governments?” International public management journal, v. 13, n. 4, pp. 350-380.

https://doi.org/10.1080/10967494.2010.524834

Sandoval, Rodrigo (2017), "Gobierno abierto: conceptos, cifras y futuros". En: Gil-García, José-Ramón; Criado-Grande, Ignacio. Tecnologías de información y comunicación en la administración pública: Conceptos, enfoques, aplicaciones y resultados. México: Infotec, pp. 293-317. ISBN: 9786077763246

https://www.researchgate.net/publication/316552519_Gobierno_Abierto_conceptos_cifras_y_futuros

Susha, Iryna; Zuiderwijk, Anneke; Janssen, Marijn; Grönlund, Åke. (2014). “Benchmarks for evaluating the progress of open data adoption: Usage, limitations, and lessons learned". Social science computer review, v. 33, n. 5, pp. 613-630. https://doi.org/10.1177/0894439314560852

Thorsby, Jeffrey; Stowers, Genie N. L.; Wolslegel, Kristen; Tumbuan, Ellie (2017). "Understanding the content and features of open data portals in American cities". Government information quarterly, v. 34, n. 1, pp. 53-61. https://doi.org/10.1016/j.giq.2016.07.001

Ubaldi, Barbara (2013). "Open government data: Towards empirical analysis of open government data initiatives". OECD Working papers on public governance, n. 22. Paris: OECD Publishing.

https://doi.org/10.1787/19934351 


\section{Anexo. Items y criterios de codificación}

Cada ítem se ha valorado con 1 punto en caso de estar presente o 0 en caso contrario, a excepción de los 3 primeros items de la dimensión Catálogo de datos.

\begin{tabular}{|c|c|}
\hline Catálogo de datos & Estudios previos \\
\hline $\begin{array}{l}\text { Número de conjuntos de datos }(0,1 \text { x número de conjuntos de datos, con un } \\
\text { máximo de } 20)^{*}\end{array}$ & $\begin{array}{l}\text { Carrasco y Sobrepere (2015); Medina (2015); Chatfield y } \\
\text { Reddick (2017); Thorsby et al. (2017) }\end{array}$ \\
\hline Número de categorías (1 a 4: 1 punto; 5 a 9: 2 puntos; 10 ó más: 3 puntos)** & Thorsby et al. (2017) \\
\hline Datos en formato legible por máquina ( 5 si se tiene, 0 en caso contrario) & Máchová y Lněnička (2017); Thorsby et al. (2017) \\
\hline Explicación de los metadatos utilizados & Lourenço (2015); Thorsby et al. (2017) \\
\hline Se indica la fecha de la última actualización de datos & Lourenço (2015); Medina (2015); Máchová y Lněnička (2017) \\
\hline Se indica el número de descargas de cada conjunto de datos & Carrasco y Sobrepere (2015); Máchová y Lněnička (2017) \\
\hline Contenido del portal & Estudios previos \\
\hline Apartado de novedades & Medina (2015) \\
\hline Demostrador & Thorsby et al. (2017) \\
\hline Lista de preguntas frecuentes (FAQs) & Máchová y Lněnička (2017) \\
\hline Tutoriales & Thorsby et al. (2017) \\
\hline Ejemplos de aplicaciones desarrolladas & $\begin{array}{l}\text { Lourenço (2015); Medina (2015); Máchová y Lněnička (2017); } \\
\text { Thorsby et al. (2017) }\end{array}$ \\
\hline APIs para desarrolladores & $\begin{array}{l}\text { Medina (2015); Chatfield y Reddick (2017); Thorsby et al. } \\
\text { (2017) }\end{array}$ \\
\hline Estadísticas de uso & $\begin{array}{l}\text { Carrasco y Sobrepere (2015); Chatfield y Reddick (2017), } \\
\text { Medina (2015) }\end{array}$ \\
\hline Política de datos abiertos & $\begin{array}{l}\text { Medina (2015); Chatfield y Reddick (2017); Thorsby et al. } \\
(2017)\end{array}$ \\
\hline Licencia para la utilización de datos & Medina (2015); Máchová y Lněnička (2017) \\
\hline Tiene algún certificado & - \\
\hline Accesibilidad y visualización & Estudios previos \\
\hline Sección de ayuda & Máchová y Lněnička (2017); Thorsby et al. (2017) \\
\hline Posibilidad de crear gráficos online & $\begin{array}{l}\text { Chatfield y Reddick (2017); Máchová y Lněnička (2017); } \\
\text { Thorsby et al. (2017) }\end{array}$ \\
\hline Posibilidad de manipular datos online & Chatfield y Reddick (2017); Thorsby et al. (2017) \\
\hline Organización por categorías & Máchová y Lněnička (2017); Thorsby et al. (2017) \\
\hline Posibilidad de ordenar los datos en el catálogo según otros criterios & Máchová y Lněnička (2017); Lourenço (2015) \\
\hline Datos clasificados por estrellas según el grado de apertura & Medina (2015) \\
\hline Buscador & $\begin{array}{l}\text { Lourenço (2015); Medina (2015); Máchová y Lněnička (2017); } \\
\text { Thorsby et al. (2017) }\end{array}$ \\
\hline Participación ciudadana & Estudios previos \\
\hline Posibilidad de sugerir nuevos conjuntos de datos & $\begin{array}{l}\text { Lourenço (2015); Máchová y Lněnička (2017); Thorsby et al. } \\
\text { (2017) }\end{array}$ \\
\hline Posibilidad de enviar comentarios o ideas de cómo mejorar el servicio & Lourenço (2015), Máchová y Lněnička (2017) \\
\hline Posibilidad de valorar los conjuntos de datos & Lourenço (2015); Máchová y Lněnička (2017) \\
\hline Información completa de contacto & Medina (2015); Thorsby et al. (2017) \\
\hline Concursos o actividades para promover el uso de los datos & Chatfield y Reddick (2017); Thorsby et al. (2017) \\
\hline
\end{tabular}

\section{Notas:}

* Valorar los conjuntos de datos ponderándolos por el número de habitantes de cada ciudad (tal como hacen Thorsby et al., 2017) desvirtuaba los resultados. Las ciudades más pobladas -como Madrid, con más de 3.000 .000 de habitantes-resultaban infravaloradas: por muchos conjuntos de datos que tuviesen, al ponderar los conjuntos de datos por cada 100.000 habitantes, el resultado era muy bajo.

** Aunque no se valoró de forma específica la utilización de estándares para la categorización de los conjuntos de datos, los portales que utilizan los estándares NTI-RISP (Norma técnica de interoperabilidad de reutilización de recursos de la información, resolución 19 de febrero de 2013,21 categorías) o DCAT-AP (Application profile for data portals in Europe, 14 categorías), véase Abella, Ortiz-de-Urbina-Criado y De-Pablos-Heredero (2017b), fueron valorados con la máxima puntuación, al contar con más de 10 categorías. 